\title{
THE ADIABATIC LIMIT FOR MULTIDIMENSIONAL HAMILTONIAN SYSTEMS
}

\author{
MAURICE A. DE GOSSON
}

Communicated by Gijs Tuynman

\begin{abstract}
We study some properties of multidimensional Hamiltonian systems in the adiabatic limit. Using the properties of the Poincaré-Cartan invariant we show that in the integrable case conservation of action requires conditions on the frequencies together with conservation of the product of energy and period. In the ergodic case the most general conserved quantity is not volume but rather symplectic capacity; we prove that even in this case there are periodic orbits whose actions are conserved.
\end{abstract}

\section{Introduction}

Our purpose is to study some properties of Hamiltonian dynamics in the adiabatic limit for systems with an arbitrary number of degrees of freedom under certain conditions on the initial and final frequencies.

Put expeditiously, the adiabatic limit is the limit of slow change of some timedependent parameters. For instance, the adiabatic limit of the motion of a pendulum with variable length and/or mass is the (ideal) motion of this pendulum when the rate of change of the frequency becomes "infinitely small". The adiabatic limit does usually not coincide with the limit of the dynamics obtained by "freezing" the parameter (if the parameters are kept constant, energy is conserved, while it is usually not in the adiabatic limit, cf. the pendulum). While the adiabatic invariance of Hamiltonian systems with one degree of freedom is well understood (see for instance Arnold's paper [2] for a thorough discussion), the case of multidimensional systems is far from being understood. This is due to the fact that the traditional approaches to the study of adiabatic invariance make use, at one moment or another, of an averaging procedure (i.e. a first order perturbation calculation), and such perturbation methods usually fail when there are several degrees of freedom. For a lucid discussion of the difficulties which appear 
when one tries to average over more than one angle see Verhulst [29] and [23] where some interesting mechanical systems are studied using averaging methods. We will show that:

- In the completely integrable case the passage to the adiabatic limit is not sufficient to guarantee conservation of action; one needs for that additional assumptions on the initial and final periods; under these assumptions, conservation of action is equivalent to the conservation of the product energy $\times$ period.

- In the ergodic case, the most natural candidates for adiabatic invariance are the symplectic capacities of the interior of the energy shell. These are better invariants than volume for the following two reasons: first, a symplectic capacity can have a finite value even if the volume is infinite; a second reason is that there exists a privileged symplectic capacity, the Hofer-Zehnder capacity, which is closely related to the notion of action of periodic orbits.

The main results of symplectic topology that we need in this article are reviewed in an Appendix (Section 6).

Notations and Basic Assumptions. We will use indifferently the notations $(x, p)$ and $z$ for points in phase space. The phase space $\mathbb{R}_{z}^{2 n}=\mathbb{R}_{x}^{n} \times \mathbb{R}_{p}^{n}$ is endowed with the standard symplectic form

$$
\sigma=\mathrm{d} p \wedge \mathrm{d} x=\sum_{j=1}^{n} \mathrm{~d} p_{j} \wedge \mathrm{d} x_{j}
$$

and we denote by $J$ the standard symplectic matrix of order $2 n$

$$
J=J=\left(\begin{array}{cc}
0_{n} & I_{n} \\
-I_{n} & 0_{n}
\end{array}\right) .
$$

The euclidean scalar product on any space $\mathbb{R}^{m}$ will be denoted by brackets $\langle\cdot, \cdot\rangle$, and the associated norm by $|\cdot|$.

The extended (time-dependent) phase space is $\mathbb{R}_{z, t}^{2 n+1}=\mathbb{R}_{z}^{2 n} \times \mathbb{R}_{t}$. For a function $H \in C^{\infty}\left(\mathbb{R}_{z, t}^{2 n+1}, \mathbb{R}\right)$ the associated suspended Hamiltonian vector field is defined by $\tilde{X}=\left(J \partial_{z} H, 1\right)$; its projection on $x, p$ space is the usual Hamiltonian vector field $X=J \partial_{z} H$. The trajectories of $\tilde{X}$ are the solutions $t \longmapsto(z(t), t)$ of the differential system

$$
\dot{z}(t)=J \partial_{z} H(z(t), t), \quad \dot{t}=1 .
$$


We will denote by $\tilde{f}_{t}$ the flow of $\tilde{X}$ (it is the suspended Hamiltonian flow determined by $H$ ), and we define the associated time-dependent flow $f_{t, t^{\prime}}$ by

$$
\left(f_{t, t^{\prime}}\left(z^{\prime}\right), t\right)=\tilde{f}_{t-t^{\prime}}\left(z^{\prime}, t^{\prime}\right) .
$$

Notice that Chapman-Kolmogorov's law holds for $f_{t, t^{\prime}}$

$$
f_{t, t^{\prime}} \circ f_{t^{\prime}, t^{\prime \prime}}=f_{t, t^{\prime \prime}}, \quad f_{t, t}=I_{d} .
$$

(we will always make the simplifying - but inessential - assumption that the flows are defined for all times). The Poincaré-Cartan invariant one-form will be denoted by $\tilde{\alpha}_{H}$. Let us recall that

$$
\tilde{\alpha}_{H}=\langle p, \mathrm{~d} x\rangle-H \mathrm{~d} t .
$$

The adjective "invariant" comes from the fact that $\tilde{\alpha}_{H}$ is a relative integral invariant of the suspended Hamiltonian field $\tilde{X}_{H}$, that is

$$
i_{\tilde{X}_{H}} \mathrm{~d} \tilde{\alpha}_{H}=0
$$

where $i_{\tilde{X}_{H}}$ is the contraction operator with $\tilde{X}_{H}$ (see [22]). A fundamental consequence of (4) is the following: let $\tilde{\gamma}$ be a curve in extended phase space, and $\mathcal{S}$ the surface in extended phase space swept out by $\left(\tilde{f}_{t}\right)$. Let $\delta$ be any closed curve on $\mathcal{S}$. Then

$$
\int_{\delta} \tilde{\alpha}_{H}=0
$$

(see for instance [12]).

\section{Definition of the Adiabatic Limit}

Let $H(\cdot, \lambda)$ be a family of Hamiltonians depending in a $C^{\infty}$ fashion on a multiparameter $\lambda=\left(\lambda_{1}, \ldots, \lambda_{d}\right)$. It is assumed that $\lambda$ can vary in some open subset $\mathcal{D}$ of $\mathbb{R}^{d}(d \geq 1)$. When $\lambda$ is allowed to depend on time, the function $t \longmapsto \lambda(t)$ describes a curve in $\mathcal{D}$ and the family $H(\cdot, \lambda)$ has thus become a time-dependent Hamiltonian $H(\cdot, \lambda(t))$. In all what follows it is assumed that $\lambda=\lambda(t)$ is constant outside an interval $[0, T](T>0)$, so that for times $t \leq 0$ or $t \geq T$ Hamilton's equations for $H(\cdot, \lambda(t))$ form an autonomous differential system. The sets

$$
\Sigma_{\text {in }}=\left\{z ; H(z, \lambda(0))=E_{\text {in }}\right\}, \quad \Sigma_{\text {fin }}=\left\{z ; H(z, \lambda(T))=E_{\text {fin }}\right\}
$$

will be called the "initial and final energy shells". The sets bounded by these shells are

$$
\Omega_{\text {in }}=\left\{z ; H(z, \lambda(0)) \leq E_{\text {in }}\right\}, \quad \Omega_{\text {fin }}=\left\{z ; H(z, \lambda(T)) \leq E_{\text {fin }}\right\} .
$$




\subsection{Completely Integrable Systems}

Assume that each of the Hamiltonians $H(\cdot, \lambda)$ is integrable: there exists a canonical change of variables $g_{\lambda}:(\phi, I) \longmapsto(x, p)$ (generally depending on $\lambda$ ) such that for fixed $\lambda$ we have

$$
H(z, \lambda)=\left(H\left(g_{\lambda}(\phi, I), \lambda\right)=K^{0}(I, \lambda) .\right.
$$

Since $g_{\lambda}$ is canonical we have

$$
d(\langle p, \mathrm{~d} x\rangle+\langle\phi, \mathrm{d} I\rangle)=\mathrm{d} p \wedge \mathrm{d} x-\mathrm{d} I \wedge \mathrm{d} \phi=0
$$

hence there exists $S_{\lambda}=S(x, I ; \lambda)$ such that

$$
\langle p, \mathrm{~d} x\rangle+\langle\phi, \mathrm{d} I\rangle=\mathrm{d} S_{\lambda}
$$

for fixed $\lambda$, that is, the function $S_{\lambda}$ is a generating function for $g_{\lambda}$

$$
(x, p)=g_{\lambda}(\phi, I) \Longleftrightarrow \phi=\partial_{I} S_{\lambda}, \quad p=\partial_{x} S_{\lambda} .
$$

An immediate calculation shows that in the $(I, \phi)$ coordinates the Poincaré-Cartan invariant (3) is given by

$$
\tilde{\alpha}_{H}=\langle I, \mathrm{~d} \phi\rangle-\left(K^{0}+\partial_{t} S_{\lambda}\right) \mathrm{d} t-\mathrm{d}\left(\langle\phi, I\rangle-S_{\lambda}\right)
$$

where $K^{0}(\cdot, \lambda)$ only depends on the action variables $I$. The solutions of Hamilton's equations in the $(\phi, I)$ variables are hence determined by the Hamiltonian $K=K^{0}+\partial_{t} S_{\lambda}$, that is

$$
K(\phi, I ; \lambda)=K^{0}(I ; \lambda)+\left\langle\partial_{\lambda} S(x(\phi, I), I ; \lambda), \dot{\lambda}\right\rangle .
$$

Definition 1. The "passage to the adiabatic limit" for the integrable system with time-dependent Hamiltonian $H(\cdot, \lambda(t))$ consists in replacing $K(\phi, I ; \lambda(t))$ by $K^{0}(I ; \lambda(t))$.

The motivation for this definition is the following: if the change of the multiparameter $\lambda$ is very slow, then the term between brackets in (7) can be neglected, and one is thus inclined to believe that it is a good approximation to replace the "true" Hamiltonian $K=K(\phi, I ; \lambda)$ by $K^{0}=K^{0}(I ; \lambda)$ (the legitimacy of this procedure is a problem belonging to the theory of differential equations, and will not be investigated here). The argument is easily made plausible by the example of the harmonic oscillator with Hamiltonian

$$
H=\sum_{j=1}^{n} \frac{1}{2 m_{j}}\left(p_{j}^{2}+m_{j}^{2} \omega_{j}^{2} x_{j}^{2}\right)
$$


where the multi-parameter is $\lambda=\left(\omega_{1}, \ldots, \omega_{n}\right)$. The canonical change of variables defined by

$$
x_{j}=\sqrt{2 I_{j} / m_{j} \omega_{j}} \cos \theta_{j} \quad \text { and } \quad p_{j}=\sqrt{2 I_{j} m_{j} \omega_{j}} \sin \theta_{j}
$$

for $1 \leq j \leq n$ brings $H$ into the normal form

$$
K^{0}(I, \lambda)=\sum_{j=1}^{n} \omega_{j} I_{j}
$$

If the time-dependence of $\lambda$ is implemented, transforming back the solutions of Hamilton's equations for (8) does not yield the solutions for $H$ - one has instead to solve those for

$$
K(I, \phi, \lambda(t))=K^{0}(I, \lambda)+\sum_{j=1}^{n} \frac{\dot{\omega}_{j}}{2 \omega_{j}} \sin 2 \phi_{j}
$$

before returning to the $x, p$. It is easy to show, using standard estimates, that if the ratios $\dot{\omega}_{j} / \omega_{j}$ are small, e.g. $\left|\dot{\omega}_{j} / \omega_{j}\right| \leq \varepsilon \ll 1$, then the error committed when solving Hamilton's equations for $K^{0}$ instead of those for $K$ is $O(\varepsilon)$ on the timescale $0 \leq t \leq 1 / \varepsilon$. Observe that formula (9) illustrates the fact that one has to assume in most cases that the frequencies do not vanish anywhere (see however the example of the linear coupled oscillator in Subsection 4.2)

An important property is that in the adiabatic limit initial Lagrangian tori are transformed into final Lagrangian tori by the flow. Making use of the terminology proposed by Weinstein [30] the path followed by an initial torus is called an "isodrast".

Proposition 2. In the adiabatic limit a torus $\mathbb{V}_{\text {in }} \subset \Sigma_{\text {in }}$ is transformed by the flow $f_{t, t^{\prime}}$ into a torus $\mathbb{V}_{\text {fin }} \subset \Sigma_{\text {fin }}$.

$$
\mathbb{V}_{T}=f_{T, 0}\left(\mathbb{V}_{\text {in }}\right)=\mathbb{V}_{\text {fin }} .
$$

Proof: The solutions of Hamilton's equations for $K^{0}$ are

$$
I(t)=I\left(t_{0}\right), \quad \phi(t)=\phi\left(t_{0}\right)+\int_{t_{0}}^{t} \omega\left(I\left(t_{0}\right), s\right) \mathrm{d} s
$$

where the frequency vector $\omega=\left(\omega_{1}, \ldots, \omega_{n}\right)$ is defined by the usual formula

$$
\omega(I, t)=\partial_{I} K^{0}(I, \lambda(t))
$$


For $t \leq 0$ the motion is thus given by

$$
I(t)=I(0), \quad \phi(t)=\phi(0)+\omega(0) t
$$

$(\omega(0)=\omega(I(0), 0))$ and for $t \geq T$ by

$$
I(t)=I(0), \quad \phi(t)=\phi(0)+\phi_{\mathcal{H}}(T)+\omega(T)(t-T)
$$

$(\omega(T)=\omega(I(0), T))$, where

$$
\phi_{\mathcal{H}}(T)=\int_{0}^{T} \omega(t) \mathrm{d} t .
$$

Choose an arbitrary point

$$
\left(I\left(t_{0}\right), \phi\left(t_{0}\right)\right)=\left(I(0), \phi(0)+\omega(0) t_{0}\right) \in \mathbb{V}_{\text {in }} .
$$

Setting $\omega(t)=\omega(I(0), t)$ the image of that point by $f_{T, 0}$ is

$$
\begin{aligned}
f_{T, 0}\left(I\left(t_{0}\right), \phi\left(t_{0}\right)\right) & =\left(I\left(t_{0}\right), \phi\left(t_{0}\right)+\int_{t_{0}}^{T} \omega(s) \mathrm{d} s\right) \\
& =\left(I(0), \phi(0)+\int_{0}^{T} \omega(s) \mathrm{d} s\right)
\end{aligned}
$$

hence $f_{T, 0}\left(I\left(t_{0}\right), \phi\left(t_{0}\right)\right) \in \mathbb{V}_{\text {fin }}$ and hence $\mathbb{V}_{\text {fin }}=f_{T, 0}\left(\mathbb{V}_{\text {in }}\right)$.

The following question will be addressed in Section 3:

Under which assumptions on the Hamiltonian $H(\cdot, \lambda)$ do the periodic orbits $\gamma_{\text {in }}$ and $\gamma_{\mathrm{fin}}$ have the same action in the adiabatic limit?

We will discuss the answer(s) to this question in Theorem 9 of Section 3.

\subsection{Ergodic Hamiltonian Systems}

In the completely integrable case each motion (periodic or not) forever winds around a given Lagrangian torus belonging to the Lagrangian foliation of the energy shell. Lagrangian tori being submanifolds with measure zero of the energy shell, any particular motion is thus confined to a very small region of the energy shell. This is in strong contrast with the generic non-integrable case where the motion is chaotic, and where every point wanders ergodically over its own energy shell. We remark, for later use (Subsection 4.1), that periodic orbits may however exist on the energy shell, independently of the existence of so-called "islands of 
stability"; this does not contradict ergodicity, since the system does not reach all the points of the energy shell in finite time. The question that poses itself is then whether there exists some quantity which could be a good candidate for adiabatic conservation. A classical heuristic argument is the following: as time-dependence on $\lambda$ is implemented, an ensemble of points on $\Sigma_{\text {in }}$ will qualitatively have similar trajectories, and in particular have approximately the same energies if the change of the parameter $\lambda$ is small. Thus, if the change of $\lambda$ is "infinitely slow" an initial ensemble located on $\Sigma_{\text {in }}$ will evolve after time $T$ into a final ensemble located on $\Sigma_{\text {fin. }}$. This heuristic observation motivates the following definition:

Definition 3. The "passage to the ergodic adiabatic limit" for chaotic (ergodic) Hamiltonian systems consists in assuming that every point $z_{0} \in \Sigma_{\text {in }}$ becomes after time $T$ a point $z_{T}=f_{T, 0}\left(z_{0}\right) \in \Sigma_{\text {fin }}$ for every $z_{0} \in \Sigma_{\text {in }}$.

In the ergodic adiabatic limit the initial energy shell is thus transformed into the final energy shell by the flow

$$
\Sigma_{\mathrm{fin}}=\Sigma_{T}=f_{T, 0}\left(\Sigma_{\mathrm{in}}\right) .
$$

In view of Liouville's theorem the phase space volume enclosed by the energy shell is invariant in the ergodic adiabatic limit; the consequences of this fact have been explored by many authors, e.g. [3, 8, 26]. It turns out that there are, as was hinted at in the introduction, more general quantities which are excellent candidates for adiabatic conservation. These quantities are the symplectic capacities of the interior of the energy shells (the notion of symplectic capacity is reviewed in the Appendix). In fact:

Proposition 4. Let $\Omega_{\text {in }}$ (respectively $\Omega_{\text {fin }}$ ) be the set bounded by the energy shell $\Sigma_{\text {in }}\left(\right.$ resp. $\left.\Sigma_{\text {fin }}\right)$. In the ergodic adiabatic limit we have

$$
c\left(\Omega_{\text {fin }}\right)=c\left(\Omega_{\text {in }}\right)
$$

for every symplectic capacity c on $\mathbb{R}_{z}^{2 n}$.

Proof: All symplectic capacities are symplectic invariants, i.e. they are preserved by canonical transformations. In particular

$$
c\left(\Omega_{\text {fin }}\right)=c\left(f_{T, 0}\left(\Omega_{\text {in }}\right)\right)=c\left(\Omega_{\text {in }}\right) .
$$

An immediate consequence of (13) is: 
Corollary 5. The area $\pi R^{2}$ of the big circle of the largest phase space ball that can be squeezed inside $\bar{\Omega}=\{z ; H(z, \lambda) \leq E\}$ is constant in the ergodic adiabatic limit.

Proof: The area $\pi R^{2}$ is a symplectic capacity, namely the "Gromov width" of $\bar{\Omega}$ (see formula (34) in the Appendix).

The following questions will be answered in Section 4:

Is there a periodic orbit on $\Sigma_{\mathrm{in}}$ which ends into a periodic orbit on $\Sigma_{\mathrm{fin}}$ having the same action? Is there a relation between the initial and final energies?

We will see that the answer to both questions is "yes".

\section{Study of the Integrable Case}

It will be assumed throughout this Section that the initial energy shell $\Sigma_{\text {in }}$ carries a periodic orbit $\gamma_{\text {in }}$ (this statement is independent of the choice of Hamiltonian; see Proposition 16 of the Appendix). This is the case for instance if $\Omega_{\text {in }}$ is compact and convex, but this is indeed not a necessary condition because one can show that almost every energy shell carries periodic orbits (see for instance [18]). Let $t \longmapsto z(t)$ be the trajectory of a point situated on $\gamma_{\text {in }}$ at time $t=0$. That point will in general leave the hypersurface $\Sigma_{\text {in }}$ and travel trough phase space, but since the Hamiltonian becomes anew time-independent for $t \geq T$, it will remain on $\Sigma_{\mathrm{fin}}$ after time $T$. We make the assumption that the motion again becomes periodic; through $z(T)$ thus passes a periodic orbit $\gamma_{\mathrm{fin}}$ of $H(z, \lambda(T))$, lying on the final energy shell $\Sigma_{\text {fin }}$.

Let us investigate under which conditions we have

$$
\oint_{\gamma_{\text {in }}}\langle p, \mathrm{~d} x\rangle=\oint_{\gamma_{\text {fin }}}\langle p, \mathrm{~d} x\rangle
$$

in the adiabatic limit. We begin by discussing some conditions on the initial and final frequencies.

\subsection{Conditions on the Frequencies}

What do these considerations imply for the initial and final periodic orbits? Since $\gamma_{\text {in }}$ is a periodic orbit lying on the torus $\mathbb{V}_{\text {in }}$ it is described in terms of the angleaction variables by the formulas

$$
I_{j}(t)=I_{j}(0), \quad \phi_{j}(t)=\phi_{j}(0)+\omega_{j}(0) t, \quad-\tau_{\text {in }} \leq t \leq 0 .
$$


In order that these relations effectively describe a periodic motion, we impose the following resonance condition:

Condition 6. (Initial Resonance) Let $\mathcal{J}_{\text {in }}$ be the set of integers $j$ for which $I_{j}(t) \neq$ 0 and $\omega_{j}(0)>0$. We assume that there exists $j_{\text {in }} \in \mathcal{J}_{\text {in }}$ such that all the frequencies $\omega_{j}(0)$ are proportional to $\omega_{j_{i n}}(0)$.

Denoting that fundamental frequency $\omega_{j_{\text {in }}}(0)$ by $\omega_{\text {in }}$ the orbit $\gamma_{\text {in }} \subset \mathbb{V}_{\text {in }}$ thus has period $\tau_{\text {in }}=2 \pi / \omega_{\text {in }}$. Similarly:

Condition 7. (Final Resonance) Let $\mathcal{J}_{\text {fin }}$ be the set of integers $j$ for which $I_{j}(t) \neq$ 0 and $\omega_{j}(T)>0$. We assume that there exists $j_{\text {fin }} \in \mathcal{J}_{\text {fin }}$ such that all the frequencies $\omega_{j}(0)$ are proportional to $\omega_{j_{\text {fin }}}(0)$.

The orbit $\gamma_{\text {fin }} \subset \mathbb{V}_{\text {fin }}$ then has period $\tau_{\text {fin }}=2 \pi / \omega_{\text {fin }}$ where $\omega_{\text {fin }}=\omega_{j_{\text {fin }}}(0)$.

We will assume in the sequel that $\mathcal{J}=\{1, \ldots, k\}(k \leq n)$; up to a reordering of the coordinates, this is of course no restriction.

Are these two conditions sufficient to ensure exact (or adiabatic) conservation of the actions of the initial and final periodic orbits $\gamma_{\text {in }}$ and $\gamma_{\text {fin }}$ ? No, unless $n=1$, in which case the orbits coincide with the Lagrangian tori since the latter are simply the circles carrying these orbits. This is immediately seen as follows. The initial resonance condition implies that

$$
\begin{aligned}
\oint_{\gamma_{\text {in }}}\langle I, \mathrm{~d} \phi\rangle & =\sum_{j=1}^{k} I_{j}(0) \int_{-\tau_{\text {in }}}^{0} \omega_{j}(s) \mathrm{d} s \\
& =\sum_{j=1}^{k} I_{j}(0) \omega_{j}(0) \tau_{\text {in }} \\
& =\sum_{j=1}^{k} 2 \pi k_{j} I_{j}(0) .
\end{aligned}
$$

Similarly, the final resonance condition leads to

$$
\oint_{\gamma_{\text {in }}}\langle I, \mathrm{~d} \phi\rangle=\sum_{j=1}^{k} 2 \pi k_{j}^{\prime} I_{j}(0)
$$

hence we will have equality of the initial and final actions if $k_{j}=k_{j}^{\prime}$ (the condition is of course not necessary). We will henceforth always make the following assumption of proportionality on the initial and final frequencies: 
There exists a constant $\rho(T)>0$ such that we have

$$
\omega_{j}(T)=\rho(T) \omega_{j}(0) \text { for } j \in \mathcal{J}
$$

that is

$$
\left(\omega_{1}(T), \ldots, \omega_{k}(T)\right)=\rho(T)\left(\omega_{1}(0), \ldots, \omega_{k}(0)\right)
$$

Notice that this assumption holds in particular when $\omega(T)=\omega(0)$, i.e. in the case of a cyclic evolution where $\lambda$ describes a loop in parameter space.

We emphasize that both the resonance conditions and the proportionality assumption above are about the initial and final motions only. No assumption whatsoever is made on the intermediary frequencies.

Remark 8. Condition (14) implies that the Maslov indices of $\gamma_{\text {in }}$ and $\gamma_{\text {fin }}$ are equal: by definition these Maslov indices are

$$
m\left(\gamma_{\text {in }}\right)=2 \sum_{j} k_{j}, \quad m\left(\gamma_{\text {fin }}\right)=2 \sum_{j} k_{j}^{\prime} .
$$

Notice that the converse is not generally true: the equality $m\left(\gamma_{\text {in }}\right)=m\left(\gamma_{f i n}\right)$ does not imply (14).

This remark is useful in the context of "adiabatic switching", where one tries to find the energy of a "deformed" Hamiltonian in terms of a known (integrable) one.

\subsection{Adiabatic Limit and Energy}

In many physics textbooks one often establishes the adiabatic invariance of the action variable for the Hamiltonian

$$
H=\frac{1}{2}\left(p^{2}+\omega^{2}(t) x^{2}\right)
$$

by the following argument. Under the assumption that the frequency changes "infinitely slowly" in the time interval $[0, T]$, one proves that the ratio $E(t) / \omega(t)$ between the instantaneous energy and frequency is conserved during the motion (this property is in fact obvious, passing to angle-action variables in the adiabatic limit). Now, the initial and final periodic orbits are the ellipses

$$
\frac{1}{2 E(0)}\left(p^{2}+\omega^{2}(0) x^{2}\right)=1 \quad \text { and } \quad \frac{1}{2 E(T)}\left(p^{2}+\omega^{2}(T) x^{2}\right)=1
$$


respectively. The areas of these ellipses are identical with the initial and final actions; since these areas are $2 \pi E(0) / \omega(0)$ and $2 \pi E(T) / \omega(T)$ respectively, one concludes to the conservation of action.

We are going to use the invariance property (4) of the Poincaré-Cartan form (3) to show that this relation between initial and final actions and initial and final energies persists for multidimensional systems. (We mention that the properties of the Poincaré-Cartan invariant have been used in [7] to establish results of adiabatic invariance for quite general systems of linear differential equations.)

Let us state and prove the main result of this Section:

Theorem 9. Assume that each $H(\cdot, \lambda)$ is integrable and that there exists a canonical change of variables $g_{\lambda}$ such that

$$
H\left(g_{\lambda}(z), \lambda\right)=K(I, \lambda) \quad \text { for every } \quad \lambda \in \mathcal{D} .
$$

Let $E_{\text {in }}=H(z(0), \lambda(0))$ and $E_{f i n}=H(z(T), \lambda(T))$ be the initial and final energies, and $\tau_{\mathrm{in}}, \tau_{\mathrm{fin}}$ the periods of $\gamma_{\mathrm{in}}$ and $\gamma_{\mathrm{fin}}$. If the proportionality assumption (14) holds, then

$$
\oint_{\gamma_{\text {in }}}\langle p, \mathrm{~d} x\rangle-\oint_{\gamma_{\text {fin }}}\langle p, \mathrm{~d} x\rangle=E_{\text {in }} \tau_{\text {in }}-E_{\text {fin }} \tau_{\text {fin }}
$$

and hence

$$
\oint_{\gamma_{\text {in }}}\langle p, \mathrm{~d} x\rangle=\oint_{\gamma_{\text {fin }}}\langle p, \mathrm{~d} x\rangle \Longleftrightarrow E_{\text {in }} \tau_{\text {in }}=E_{\text {fin }} \tau_{\text {fin }} .
$$

Proof: Let us denote by $t \longmapsto z(t)$ the trajectory carrying the periodic orbits $\gamma_{\text {in }}$ and $\gamma_{\text {fin. }}$. The integrability assumption implies that for time $t \leq 0$ the motion takes place on an initial Lagrangian torus $\mathbb{V}_{\text {in }} \subset \Sigma_{\text {in }}$ and for time $t \geq T$ on a final torus $\mathbb{V}_{\text {fin }} \subset \Sigma_{\text {fin }}$. We lift the periodic orbits $\gamma_{\text {in }}$ and $\gamma_{\text {fin }}$ to curves

$$
\tilde{\gamma}_{\text {in }}:\left[-\tau_{\text {in }}, 0\right] \longrightarrow \mathbb{R}_{z, t}^{2 n+1} \text { and } \quad \tilde{\gamma}_{\text {fin }}(t):\left[T, T+\tau_{\text {fin }}\right] \longrightarrow \mathbb{R}_{z, t}^{2 n+1}
$$

in extended phase space, defined by

$$
\tilde{\gamma}_{\text {in }}(t)=\left(\gamma_{\text {in }}(t), t\right) \text { and } \tilde{\gamma}_{\text {fin }}(t)=\left(\gamma_{\text {fin }}(t), t\right) .
$$

We define two arcs $\tilde{\xi}_{\text {in }}$ and $\tilde{\xi}_{\text {fin }}$ in $\mathbb{R}_{z, t}^{2 n}$ as follows: $\tilde{\xi}_{\text {in }}$ is the segment of line joining $(z(0), 0)$ to $\left(z\left(-\tau_{\text {in }}\right),-\tau_{\text {in }}\right)=\left(z(0),-\tau_{\text {in }}\right)$, that is

$$
\tilde{\xi}_{\text {in }}(s)=\left(z(0),-s \tau_{\text {in }}\right), \quad 0 \leq s \leq 1
$$


and $\tilde{\xi}_{\text {fin }}$ is the (non-isochronous) deformation of $\tilde{\xi}_{\text {in }}$ defined, for $0 \leq s \leq 1$, by

$$
\tilde{\xi}_{\text {fin }}(s)=\tilde{f}_{T+(1-s) \tau_{\text {fin }}+s \tau_{\text {in }}}\left(z(0),-s \tau_{\text {in }}\right)
$$

that is, by definition of the suspended flow $\tilde{f}_{t}$

$$
\tilde{\xi}_{\text {fin }}(s)=\left(f_{T+(1-s)} \tau_{\text {fin }},-s \tau_{\text {in }}(z(0)), T+(1-s) \tau_{\text {fin }}\right) .
$$

Taking into account the periodicity relations $z(0)=z\left(-\tau_{\text {in }}\right)$ and $z(T)=z(T+$ $\left.\tau_{\text {fin }}\right)$ we have

$$
\tilde{\xi}_{\text {fin }}(0)=\tilde{f}_{T+\tau_{\text {fin }}}(z(0), 0)=\left(f_{T+\tau_{\text {fin }}, 0}(z(0)), T+\tau_{\text {fin }}\right)=\left(z(T), T+\tau_{\text {fin }}\right)
$$

and

$$
\tilde{\xi}_{\text {fin }}(1)=\tilde{f}_{T+\tau_{\text {in }}}\left(z(0),-\tau_{\text {in }}\right)=\left(f_{T, 0}(z(0), T)=(z(T), T)\right.
$$

thus $\tilde{\xi}_{\text {fin }}$ is an arc joining the endpoint $\left(z(T), T+\tau_{\text {fin }}\right)$ of $\tilde{\gamma}_{\text {fin }}$ to its origin $(z(T), T)$ (in this order). Let $\Gamma$ be the surface swept out by $\tilde{\xi}_{\text {in }}$ during its travel; its boundary is the one-cycle

$$
\tilde{\gamma}=\tilde{\xi}_{\text {in }}+\tilde{\gamma}_{1}-\tilde{\xi}_{\text {fin }}-\tilde{\gamma}_{2}
$$

where $\tilde{\gamma}_{1}$ is the piece of trajectory in extended phase space joining $(z(0), 0)$ to $\left(z(T), T+\tau_{\text {fin }}\right)$ and $\tilde{\gamma}_{2}$ that joining $\left(z(0),-\tau_{\text {in }}\right)$ to $(z(T), T)$

$$
\begin{aligned}
& \tilde{\gamma}_{1}(t)=\left(f_{t, 0}(z(0)), t\right), \quad 0 \leq t \leq T+\tau_{\text {fin }} \\
& \tilde{\gamma}_{2}(t)=\left(f_{t, 0}(z(0)), t\right), \quad-\tau_{\text {in }} \leq t \leq T .
\end{aligned}
$$

The strip $\Gamma$ consists of characteristic curves of $\tilde{X}_{H}$; hence, using respectively Stokes' theorem and the consequence (5) of the relative invariance property (4) of the Poincaré-Cartan form we have

$$
\int_{\tilde{\gamma}} \tilde{\alpha}_{H}=\int_{\Gamma} \mathrm{d} \tilde{\alpha}_{H}=0
$$

that is

$$
\int_{\tilde{\gamma}_{1}} \tilde{\alpha}_{H}-\int_{\tilde{\gamma}_{2}} \tilde{\alpha}_{H}=\int_{\tilde{\xi}_{\text {fin }}} \tilde{\alpha}_{H}-\int_{\tilde{\xi}_{\text {in }}} \tilde{\alpha}_{H} \cdot
$$

By definition of the paths $\tilde{\gamma}_{1}$ and $\tilde{\gamma}_{2}$ we have

$$
\tilde{\gamma}_{1}+\tilde{\gamma}_{\text {fin }}=\tilde{\gamma}_{\text {in }}+\tilde{\gamma}_{2}
$$

where $\tilde{\gamma}_{\text {in }}(t)=\left(\gamma_{\text {in }}(t), t\right)$ for $-\tau_{\text {in }} \leq t \leq 0$ and $\tilde{\gamma}_{\text {fin }}(t)=\left(\gamma_{\text {fin }}(t), t\right)$ for $T \leq t \leq$ $T+\tau_{\text {fin }}$, hence

$$
\int_{\tilde{\gamma}_{1}} \tilde{\alpha}_{H}-\int_{\tilde{\gamma}_{2}} \tilde{\alpha}_{H}=\oint_{\gamma_{\text {in }}}\langle p, \mathrm{~d} x\rangle-\oint_{\gamma_{\text {fin }}}\langle p, \mathrm{~d} x\rangle
$$


since we have

$$
\int_{\tilde{\gamma}_{1}} \tilde{\alpha}_{H}=\int_{\tilde{\gamma}_{1}}\langle p, \mathrm{~d} x\rangle-H d t=\int_{\gamma_{1}}\langle p, \mathrm{~d} x\rangle
$$

and

$$
\int_{\tilde{\gamma}_{2}} \tilde{\alpha}_{H}=\int_{\tilde{\gamma}_{2}}\langle p, \mathrm{~d} x\rangle-H d t=\int_{\gamma_{2}}\langle p, \mathrm{~d} x\rangle .
$$

The equality (21) is thus equivalent to

$$
\oint_{\gamma_{\text {in }}}\langle p, \mathrm{~d} x\rangle-\oint_{\gamma_{\text {fin }}}\langle p, \mathrm{~d} x\rangle=\int_{\tilde{\xi}_{\text {in }}} \tilde{\alpha}_{H}-\int_{\tilde{\xi}_{\text {fin }}} \tilde{\alpha}_{H} .
$$

It follows that it is sufficient to prove that

$$
\int_{\tilde{\xi}_{\text {in }}} \tilde{\alpha}_{H}-\int_{\tilde{\xi}_{\text {fin }}} \tilde{\alpha}_{H}=E_{\text {in }} \tau_{\text {in }}-E_{\text {fin }} \tau_{\text {fin }} .
$$

We first notice that since $\langle p, \mathrm{~d} x\rangle=0$ along $\tilde{\xi}_{\text {in }}$ and that the value of the Hamiltonian along $\tilde{\xi}_{\text {in }}$ is

$$
H\left(z(0), \lambda\left(-s \tau_{\text {in }}\right)\right)=H(z(0), \lambda(0))=E_{\text {in }}
$$

we have

$$
\int_{\tilde{\xi}_{\text {in }}} \tilde{\alpha}_{H}=\int_{\tilde{\xi}_{\text {in }}} H \mathrm{~d} t=\int_{0}^{1} E_{\text {in }}\left(-\tau_{\text {in }}\right) \mathrm{d} t=E_{\text {in }} \tau_{\text {in }} .
$$

Let us next prove that

$$
\int_{\tilde{\xi}_{\text {fin }}} \tilde{\alpha}_{H}=E_{\text {fin }} \tau_{\text {fin }}
$$

the theorem will follow. Let

$$
\xi_{\text {fin }}(s)=f_{T+(1-s) \tau_{\text {fin }},-s \tau_{\text {in }}}(z(0)), \quad 0 \leq s \leq 1
$$

be the projection of $\tilde{\xi}_{\text {fin }}(s)$ on phase space $\mathbb{R}_{z}^{2 n}$ (cf. (19)). Since

$$
f_{T+(1-s) \tau_{\text {fin }},-s \tau_{\text {in }}}=f_{T+(1-s) \tau_{\text {fin }}, T} \circ f_{T, 0} \circ f_{0,-s \tau_{\text {in }}}(z(0))
$$

and $f_{0,-s \tau_{\text {in }}}\left(z(0) \in \gamma_{\text {in }}\right.$, we will have (recalling that $\mathbb{V}_{T}=\mathbb{V}_{\text {fin }}$ )

$$
\xi_{\text {fin }}(s) \in \mathbb{V}_{T}=\mathbb{V}_{\text {fin }} \subset \Sigma_{\text {fin }}
$$

hence the value of the Hamiltonian along $\tilde{\xi}_{\text {fin }}$ is

$$
H\left(\xi_{\text {fin }}(s), \lambda\left(T+(1-s) \tau_{\text {fin }}\right)\right)=H\left(\xi_{\text {fin }}(s), \lambda(T)\right)=E_{\text {fin }} .
$$


It follows that

$$
\int_{\tilde{\xi}_{\text {fin }}} H \mathrm{~d} t=-\int_{0}^{1} E_{\text {fin }}\left(-\tau_{\text {fin }}\right) \mathrm{d} t=E_{\text {fin }} \tau_{\text {fin }} .
$$

There remains to show that

$$
\oint_{\xi_{\text {fin }}}\langle p, \mathrm{~d} x\rangle=0 .
$$

Passing to angle-action variables $(\phi, I)$ and setting $z(0)=(I(0), \phi(0)), \omega(t)=$ $\omega(I(0), t)$ we have

$$
\begin{aligned}
\xi_{\text {fin }}(s) & =\left(I(0), \phi(0)+\int_{-s \tau_{\text {in }}}^{T+(1-s) \tau_{\text {fin }}} \omega(t) \mathrm{d} t\right) \\
& =\left(I(0), \phi(0)+\phi_{\mathcal{H}}(T)+s \omega(0) \tau_{\text {in }}+(1-s) \omega(T) \tau_{\text {fin }}\right) .
\end{aligned}
$$

The assumption (14) implies that we have $\omega(T) \tau_{\text {fin }}=\omega(0) \tau_{\text {in }}$ and hence

$$
\xi_{\text {fin }}(s)=\left(I(0), \phi(0)+\phi_{\mathcal{H}}(T)+\omega(T) \tau_{\text {fin }}\right)
$$

so that

$$
\oint_{\xi_{\text {fin }}}\langle p, \mathrm{~d} x\rangle=\oint_{\xi_{\text {fin }}}\langle I, \mathrm{~d} \phi\rangle=0
$$

as was to be proven.

Remark 10. The observant reader will have noticed that the proof of the identity (22) only used the fact that $\gamma_{\mathrm{in}}$ and $\gamma_{\mathrm{fin}}$ were periodic orbits carried by the initial and final energy shells. Neither integrability, nor the proportionality assumption (14), were used at this stage of the proof of Theorem 9 above. We will in fact see in the proof of Theorem 11 that integrability is not even necessary to establish the equality (25).

\section{The Ergodic Case}

We assume in this section that the Hamiltonians $H(\cdot, \lambda)$ have no other constants of the motion than the energy (for fixed $\lambda$ ). The motion is thus chaotic, and we assume that we are in the situation of the ergodic adiabatic limit. 


\subsection{Ergodic Limit and Periodic Orbits}

In the ergodic adiabatic limit, an initial energy shell uniquely determines the final energy shell. The following result is the ergodic version of Theorem 9:

Theorem 11. Assume that the initial energy shell $\Sigma_{\text {in }}$ carries a periodic orbit $\gamma_{\text {in }}$. In the ergodic adiabatic limit: i) the trajectory through any point of $\Sigma_{i n}$ becomes after time $T$ a periodic orbit $\gamma_{\text {fin }}$ of the final energy shell, and $\left.\mathrm{ii}\right)$ we have both

$$
\oint_{\gamma_{\text {in }}}\langle p, \mathrm{~d} x\rangle=\oint_{\gamma_{\text {fin }}}\langle p, \mathrm{~d} x\rangle \text { and } E_{\text {in }} \tau_{\text {in }}=E_{\text {fin }} \tau_{\text {fin }}
$$

where $\tau_{\mathrm{in}}$ and $\tau_{\mathrm{fin}}$ are the periods of $\gamma_{\mathrm{in}}$ and $\gamma_{\mathrm{fin}}$, respectively.

Proof: By definition of the ergodic adiabatic limit for any point $z_{0}$ of $\Sigma_{\text {in }}$ the final energy is given by $E_{\text {fin }}=H\left(f_{T, 0}\left(z_{0}\right), \lambda(T)\right)$, and $f_{T, 0}\left(\gamma_{\text {in }}\right) \subset \Sigma_{\text {fin }}$. Let us show $\gamma_{T}=f_{T, 0}\left(\gamma_{\mathrm{in}}\right)$ indeed is a periodic orbit of $H(\cdot, \lambda(T))$; since $f_{T, 0}\left(z_{0}\right) \in$ $f_{T, 0}\left(\gamma_{\text {in }}\right)$ this will prove part i) of the Theorem. Since in the ergodic adiabatic limit we have

$$
\Sigma_{T}=f_{T, 0}\left(\Sigma_{\text {in }}\right)=\Sigma_{\text {fin }}
$$

it follows that

$$
\Sigma_{\text {fin }}=\left\{z ; H(z, \lambda(T))=E_{\text {fin }}\right\}=\left\{u ; H\left(f_{T, 0}^{-1}(u), \lambda(0)\right)=E_{\text {in }}\right\} .
$$

In view of Proposition 16 of the Appendix the Hamiltonians $H\left(f_{T, 0}^{-1}(\cdot), \lambda(0)\right)$ and $H(\cdot, \lambda(T))$ have the same periodic orbits on $\Sigma_{\text {fin }}$. For the flow of $H\left(f_{T, 0}^{-1}(\cdot), \lambda(0)\right)$ being $f_{T, 0} \circ f_{t, 0} \circ f_{T, 0}^{-1}$ it follows that $\gamma_{T}$ is a periodic orbit of $H\left(f_{T, 0}^{-1}(\cdot), \lambda(0)\right)$, hence also of $H(\cdot, \lambda(T))$. Let us prove ii). That $\gamma_{\text {in }}$ and $\gamma_{\text {fin }}$ have same action follows immediately from the fact that $f_{T, 0}$ is canonical

$$
\oint_{\gamma_{\text {fin }}}\langle p, \mathrm{~d} x\rangle=\oint_{f_{T, 0}\left(\gamma_{\text {in }}\right)}\langle p, \mathrm{~d} x\rangle=\oint_{\gamma_{\text {in }}}\langle p, \mathrm{~d} x\rangle .
$$

To prove the second equality (26) we use Remark 10 following the proof of Theorem 9 to notice that we have

$$
\oint_{\gamma_{\text {in }}}\langle p, \mathrm{~d} x\rangle-\oint_{\gamma_{\text {fin }}}\langle p, \mathrm{~d} x\rangle=\int_{\tilde{\xi}_{\text {in }}} \tilde{\alpha}_{H}-\int_{\tilde{\xi}_{\text {fin }}} \tilde{\alpha}_{H}
$$

where $\tilde{\xi}_{\text {in }}$ and $\tilde{\xi}_{\text {fin }}$ are defined by (17), (18). In view of the equality of the actions of $\gamma_{\text {in }}$ and $\gamma_{\text {fin }}$ just established we thus have

$$
\int_{\tilde{\xi}_{\text {in }}} \tilde{\alpha}_{H}=\int_{\tilde{\xi}_{\text {fin }}} \tilde{\alpha}_{H}
$$


As in the proof of Theorem 9 we obviously have

$$
\int_{\tilde{\xi}_{\text {in }}} \tilde{\alpha}_{H}=E_{\text {in }} \tau_{\text {in }}
$$

so there remains to show that

$$
\int_{\tilde{\xi}_{\text {fin }}} \tilde{\alpha}_{H}=E_{\text {fin }} \tau_{\text {fin }}
$$

to prove the second equality (26). We have

$$
\int_{\tilde{\xi}_{\text {fin }}} \tilde{\alpha}_{H}=\oint_{\xi_{\text {fin }}}\langle p, \mathrm{~d} x\rangle-\int_{0}^{1} H\left(\xi_{\text {fin }}(s), \lambda(T)\right)\left(-\tau_{\text {fin }}\right) \mathrm{d} t
$$

where $\xi_{\text {fin }}$ is defined by

$$
\xi_{\text {fin }}(s)=f_{T+(1-s) \tau_{\text {fin }},-s \tau_{\text {in }}}\left(z_{0}\right) .
$$

Set, for $0 \leq s \leq 1$,

$$
\gamma(s)=\left\{\begin{array}{c}
f_{T,-2 s \tau_{\text {in }}}\left(z_{0}\right) \text { for } 0 \leq s \leq 1 / 2 \\
f_{T+2(1-s) \tau_{\text {fin }}, 0}\left(z_{0}\right) \text { for } 1 / 2 \leq s \leq 1
\end{array}\right.
$$

and

$$
\tilde{\gamma}(s)=\left(\gamma(s), T+(1-s) \tau_{\text {fin }}\right), \quad 0 \leq s \leq 1 .
$$

Since $\gamma(0)=\gamma(1)=f_{T, 0}\left(z_{0}\right)$ the paths $\tilde{\gamma}$ and $\tilde{\xi}_{\text {fin }}$ (see (19)) have same endpoints. On the other hand both $\tilde{\gamma}$ and $\tilde{\xi}_{\text {fin }}$ lye on the same surface $\Gamma$ swept out by $\tilde{\xi}_{\text {in }}$ during its travel. In view of the relative invariance of $\tilde{\alpha}_{H}$ we thus have

$$
\int_{\tilde{\xi}_{\text {in }}} \tilde{\alpha}_{H}=\int_{\tilde{\gamma}} \tilde{\alpha}_{H}
$$

Let us show that

$$
\int_{\tilde{\gamma}} \tilde{\alpha}_{H}=E_{\text {fin }} \tau_{\text {fin }}
$$

this will prove the second equality (26). By definition of $\tilde{\alpha}_{H}$ we have

$$
\int_{\tilde{\gamma}} \tilde{\alpha}_{H}=\oint_{\gamma}\langle p, \mathrm{~d} x\rangle-\int_{0}^{1} H(\gamma(s), \lambda(T))\left(-\tau_{\text {fin }}\right) \mathrm{d} s .
$$

Since in the ergodic adiabatic limit we have both

$$
f_{T,-2 s \tau_{\text {in }}}\left(z_{0}\right) \in \Sigma_{\text {fin }} \quad \text { and } \quad f_{T+2(1-s) \tau_{\text {fin }}, 0}\left(z_{0}\right) \in \Sigma_{\text {fin }}
$$


and hence $H(\gamma(s), \lambda(T))=E_{\text {fin }}$ for $0 \leq s \leq 1$, it follows that

$$
\int_{0}^{1} H(\gamma(s), \lambda(T))\left(-\tau_{\text {fin }}\right) \mathrm{d} s=-E_{\text {fin }} \tau_{\text {fin }} .
$$

There remains to show that we have

$$
\oint_{\gamma}\langle p, \mathrm{~d} x\rangle=0
$$

Now,

$$
f_{T,-2 s \tau_{\text {in }}}\left(z_{0}\right)=f_{T, 0} \circ f_{2 s \tau_{\text {in }}, 0}^{0}\left(z_{0}\right)
$$

where $f_{t, 0}^{0}$ is the flow of $H(\cdot, \lambda(0))$, hence

$$
s \longmapsto f_{T,-2 s \tau_{\text {in }}}\left(z_{0}\right) \quad(0 \leq s \leq 1 / 2)
$$

is a reparametrization of the loop $f_{T, 0}\left(\gamma_{\text {in }}\right)$. Similarly,

$$
f_{T+2(1-s) \tau_{\text {fin },}, 0}\left(z_{0}\right)=f_{2(1-s) \tau_{\text {fin }, 0}}^{T} \circ f_{T, 0}\left(z_{0}\right)
$$

where $f_{t, 0}^{T}$ is the flow of $H(\cdot, \lambda(T))$, hence

$$
s \longmapsto f_{T+2(1-s) \tau_{\text {fin }, 0}}\left(z_{0}\right) \quad(1 / 2 \leq s \leq 1)
$$

is a reparametrization of the opposed path of $\gamma_{\mathrm{fin}}=f_{T, 0}\left(\gamma_{\mathrm{in}}\right)$. It follows that

$$
\oint_{\gamma}\langle p, \mathrm{~d} x\rangle=\oint_{f_{T, 0}(\gamma)}\langle p, \mathrm{~d} x\rangle-\oint_{f_{T, 0}(\gamma)}\langle p, \mathrm{~d} x\rangle=0
$$

as we set out to prove.

Remark 12. Let $H$ be a time-dependent Hamiltonian. The flow $\bar{f}_{t, 0}$ of the Hamiltonian $\bar{H}$ defined by

$$
\bar{H}(z, t))=-H\left(f_{t, 0}(z), t\right)
$$

is given by $\bar{f}_{t, 0}=f_{t, 0}^{-1}$ (see [27], or [19], Proposition 1, p. 144). This Hamiltonian (up to a sign) was implicitly used in the proof of Theorem 11. Its appearance is related to the reversibility of the notion of ergodic adiabatic invariance considered here. 


\subsection{Possible Extensions}

It has been suggested (for instance in [8]) that volume might in certain cases be an adiabatic invariant for non-ergodic Hamiltonian systems. This is of course trivially untrue in most cases (to begin with $n$-dimensional oscillator). Let us briefly discuss the possibility for symplectic capacities to be adiabatic invariants. We begin with the example of a coupled linear oscillator with Hamiltonian

$$
H=\frac{1}{2}\left(p_{1}^{2}+p_{2}^{2}+\omega^{2}(t)\left(x_{1}+x_{2}\right)^{2}\right) .
$$

In the adiabatic limit we may use the canonical change of the variables $(x, p)$ to the variables $(X, P)$ defined by

$$
\begin{gathered}
\left(X_{1}, X_{2}\right)=(2 \omega(t))^{-1 / 2}\left(x_{1}-x_{2}, x_{1}+x_{2}\right) \\
\left(P_{1}, P_{2}\right)=(2 \omega(t))^{1 / 2}\left(p_{1}-p_{2}, p_{1}+p_{2}\right)
\end{gathered}
$$

to bring $H$ into the form

$$
K=\frac{\omega(t)}{2}\left(P_{1}^{2}+P_{2}^{2}+X_{1}^{2}\right)
$$

For fixed $t$ the energy shells are the hypersurfaces

$$
\Sigma: \frac{\omega}{2 E}\left(P_{1}^{2}+P_{2}^{2}+X_{1}^{2}\right)=1
$$

let $\Omega$ be the phase space region bounded by $\Sigma$. Since

$$
\frac{\omega}{2}\left(P_{1}^{2}+X_{1}^{2}\right) \leq \frac{\omega}{2}\left(P_{1}^{2}+P_{2}^{2}+X_{1}^{2}\right) \leq \frac{\omega}{2}\left(P_{1}^{2}+P_{2}^{2}+X_{1}^{2}+X_{2}^{2}\right)
$$

we have

$$
B(\sqrt{2 E / \omega}) \subset \Omega \subset Z_{1}(\sqrt{2 E / \omega})
$$

where $B(R)$ is the phase space ball centered at the origin and with radius $R$ and $Z_{1}(R)$ is the cylinder $x_{j}^{2}+p_{j}^{2} \leq R^{2}$. It follows, by (33) in the Appendix, that $c(\Omega)=2 \pi E / \omega$ for any symplectic capacity $c$. The solutions of Hamilton's equations for $K$ satisfy

$$
P_{1}^{2}(t)+P_{2}^{2}(t)+X_{1}^{2}(t)=P_{1}^{2}(0)+P_{2}^{2}(0)+X_{1}^{2}(0)
$$

for all $t$, hence the ratio between the instantaneous energy and the frequency is constant and the equality between the initial and final symplectic capacities follows. Notice that it does not make sense here to investigate whether the volume 
enclosed by the energy shell is adiabatically conserved since the volume of each $\Omega$ is infinite!

In the general case, assume that the sets $\Omega_{\text {in }}$ and $\Omega_{\text {fin }}$ bounded by the initial and final energy shells are both compact and convex. Then, in view of the second part of Theorem 15 in the Appendix, we have

$$
c_{H Z}\left(\Omega_{\text {in }}\right)=\oint_{\gamma_{\text {in }}}\langle p, \mathrm{~d} x\rangle, \quad c_{H Z}\left(\Omega_{\mathrm{fin}}\right)=\oint_{\gamma_{\mathrm{fin}}}\langle p, \mathrm{~d} x\rangle .
$$

if both $\gamma_{\text {in }}$ and $\gamma_{\text {fin }}$ are minimal periodic loops (i.e., minimizing action on their respective energy shells); $c_{H Z}$ is the Hofer-Zehnder capacity. However it is not true in general that two such minimizing periodic loops are connected by a phase space trajectory. If, however, the minimal periodic orbit $\gamma_{\text {in }}$ becomes, after time $T$, a periodic orbit $\gamma_{\text {fin }}$ carried by $\Sigma_{\text {fin }}$ and having same action, then we have

$$
\oint_{\gamma_{\text {in }}}\langle p, \mathrm{~d} x\rangle=\oint_{\gamma_{\mathrm{fin}}}\langle p, \mathrm{~d} x\rangle \geq c_{H Z}\left(\Omega_{\mathrm{fin}}\right)
$$

so that $c_{H Z}\left(\Omega_{\mathrm{in}}\right) \geq c_{H Z}\left(\Omega_{\mathrm{fin}}\right)$. If conversely a minimal periodic orbit on $\Sigma_{\mathrm{fin}}$ becomes a periodic orbit on $\Sigma_{\text {in }}$ with same action, we will also have $c_{H Z}\left(\Omega_{\text {in }}\right) \geq$ $c_{H Z}\left(\Omega_{\text {fin }}\right)$ and hence

$$
c_{H Z}\left(\Omega_{\mathrm{in}}\right)=c_{H Z}\left(\Omega_{\mathrm{fin}}\right) .
$$

\subsection{Quantization}

Let us briefly discuss semiclassical quantization from the perspective of ergodic adiabatic switching. It has been suggested by Berry $[3,5]$ that (up to asymptotic corrections) the formula

$$
\left(\frac{1}{2 \pi \hbar}\right)^{n} \int_{\Omega} \mathrm{d}^{n} x \mathrm{~d}^{n} p=N+\frac{1}{2}
$$

could provide a good quantization scheme for ergodic systems. This is, of course, perfectly in accordance with the observed adiabatic invariance of volume for such systems. Following the discussion above, it could perhaps be advantageous to replace the quantization condition (29) by its symplectic counterpart

$$
c(\Omega)=\left(N+\frac{1}{2}\right) h, \quad N=0,1,2, \ldots
$$

where $c$ is some symplectic capacity. In view of Theorem 11 this leads to the existence of quantized periodic orbits. Choose in fact $c=c_{H Z}$ in (30) and assume 
$\Omega$ compact and convex. Then in view of Theorem 15 there exists a periodic orbit $\gamma \in \Sigma=\partial \Omega$ such that

$$
\oint_{\gamma}\langle p, \mathrm{~d} x\rangle=\left(N+\frac{1}{2}\right) h .
$$

Remark 13. It would be interesting to investigate the relation between this approach and the quantization conditions for isolated periodic orbits proposed in [28].

It turns out that the conditions (30), (31) are consistent with the quantization scheme proposed in [12-14], where we made the following Ansatz:

The only admissible trajectories for a Hamiltonian $H$ in the ground energy level are those which lie on some subset (not only an energy shell) of phase space with capacity $\frac{1}{2} h$.

That Ansatz might thus be justified a posteriori using ergodic "adiabatic switching": one starts with some Hamiltonian $H_{0}$ for which one knows that this Ansatz is trivially true, and one then introduces time-dependence in order to deform $H_{0}$ into $H$. That possibility will be explored in forthcoming research.

\section{Concluding Remarks.}

We have been investigating in this article a few consequences of the passage to the adiabatic limit, both in he completely integrable and in the ergodic case. We have however not studied rigourously the conditions of validity of this limit. As we already mentioned, there are technical difficulties when one tries to use techniques from averaging theory. The solution of that problem (or at least a clue to it) might be in a systematic use of the usual comparison theorems of the theory of ordinary differential equations (Gronwall's lemma), and the improvement of existing averaging techniques. Some results for oscillating systems will be given in a forthcoming research.

A related problem is that of the technique of adiabatic switching, where one tries to calculate the (semiclassical) energy levels of a Hamiltonian $H$ in terms of those of a Hamiltonian $H_{0}$ whose energy levels are known. This procedure obviously automatically works for integrable Hamiltonians in all cases where the passage to the adiabatic limit is legitimate (the semiclassical and adiabatic limit are however not identical: see [3]). In the ergodic case one knows that the isolated periodic orbits play a fundamental role (see [16,28], and [6] for a different point of view). 
Theorem 11 can indeed be used to relate ergodic adiabatic invariance to quantization; one should however note that it says nothing about how to find periodic orbits to quantize! A related difficulty is that it is very difficult to calculate symplectic capacities outside a few textbook cases (but the same remark applies to the calculation of volumes!).

We finally notice that it would be interesting to investigate whether the study of adiabatic invariance of symplectic capacity could be done directly by adapting appropriately the techniques in the proof of Hofer and Zehnder's theorem 15 of the Appendix. The proof of this theorem (which is very difficult and technical) is based on the construction of privileged periodic Hamiltonian orbits.

\section{Appendix: Symplectic Capacities and Gromov's Theorem}

Consider a phase space ball $B(r):|x|^{2}+|p|^{2} \leq r^{2}$. The area of the projection of $B(r)$ onto any two-dimensional coordinate plane (say $x_{j}, p_{k}, x_{j}, x_{k}$ or $p_{j}, p_{k}$ ) is obviously $\pi r^{2}$. Suppose now that we move $B(r)$ using canonical transformations (e.g. a Hamiltonian flow); in view of Liouville's theorem, $B(r)$ will distort while keeping a constant volume, hence the areas of the projections on the twodimensional planes will change and a priori take arbitrary values. Not so! No matter how hard we try, the areas of the projections of the distorted ball on conjugate planes $x_{j}, p_{j}$ will never decrease; they will always be at least $\pi r^{2}$. This deep property of canonical transformations is actually equivalent to Gromov's squeezing theorem [15] (also known as the "principle of the symplectic camel"):

Theorem 14. (Gromov [15]) There exists no canonical transformation sending $B(r)$ into a phase space cylinder $Z_{j}(R): x_{j}^{2}+p_{j}^{2} \leq R^{2}$ if $r \geq R$.

Gromov's theorem allows us to define, following Ekeland and Hofer [11], the subsidiary notion of symplectic capacity: a symplectic capacity on phase space $\mathbb{R}_{z}^{2 n}$ is the assignment, to every subset $\Omega \subset \mathbb{R}_{z}^{2 n}$, of a positive number, or $+\infty$, such that the following four properties hold:

Cap1: "Monotonicity": If $\Omega \subset \Omega^{\prime}$ then $c(\Omega) \leq c\left(\Omega^{\prime}\right)$;

Cap2: “2-Homogeneity": For every $k \in \mathbb{R}$ we have $c(k \Omega)=k^{2} c(\Omega)$;

Cap3: "Symplectic invariance": If a map $f$ is a canonical transformation, then $c(f(\Omega))=c(\Omega)$. 
Cap4: "Normalization": Phase space balls and symplectic cylinders with same radii have same symplectic capacities:

$$
c(B(r))=\pi r^{2}=c\left(Z_{j}(r)\right) .
$$

Nota bene: There exist several nonequivalent definitions of the notion of symplectic capacity in the literature; see e.g. [10,19,24,27].

Notice that in view of Cap1 and Cap4 we have

$$
B(r) \subset \Omega \subset Z_{j}(r) \Longrightarrow c(\Omega)=\pi r^{2}
$$

for every symplectic capacity $c$.

A basic example of a symplectic capacity is the symplectic area (also called "Gromov's width") of a subset $\Omega \subset \mathbb{R}_{z}^{2 n}$; it is defined by

$$
c_{\mathrm{G}}(\Omega)=\sup _{f \text { canonical }}\left\{\pi r^{2} ; f(B(r)) \subset \Omega\right\} .
$$

That the properties (Cap1)-(Cap4) hold for $c_{\mathrm{G}}$ is trivial, except for the equality $c_{\mathrm{G}}\left(Z_{j}(r)\right)=\pi r^{2}$, because it is actually equivalent to Gromov's theorem, and hence highly nontrivial!

Any symplectic capacity of the ellipsoidal set defined by the inequality

$$
B\left(r_{1}, \ldots, r_{n}\right): \frac{1}{r_{1}^{2}}\left(x_{1}^{2}+p_{1}^{2}\right)+\cdots+\frac{1}{r_{n}^{2}}\left(x_{n}^{2}+p_{n}^{2}\right) \leq 1
$$

is equal to

$$
c\left(B\left(r_{1}, \ldots, r_{n}\right)\right)=c\left(Z_{j}(r)\right)=\pi r^{2}, \quad r=\inf \left(r_{1}, \ldots, r_{n}\right)
$$

It is thus the area of the smallest circle of the ellipse bounding $B\left(r_{1}, \ldots, r_{n}\right)$ (the $n$-tuple $\left(r_{1}, \ldots, r_{n}\right)$ is called the symplectic spectrum of $\left.B\left(r_{1}, \ldots, r_{n}\right)\right)$.

It turns out that there exists a privileged symplectic capacity $c_{H Z}$ (the "HoferZehnder capacity") related to the action of periodic Hamiltonian orbits:

Theorem 15. (Hofer-Zehnder [19]) There exists a symplectic capacity $c_{H Z}$ having the following properties: i) if $\Sigma_{E}: H=E$ is the boundary of a compact and convex region $\Omega_{E}$ of phase space, then

$$
\left|\oint_{\gamma}\langle p, \mathrm{~d} x\rangle\right| \geq c_{H Z}\left(\Omega_{E}\right)
$$

for every periodic orbit $\gamma$ of the Hamilton field $X_{H}$ on $\Sigma$ and: ii) there exists a periodic orbit $\gamma^{0}$ which is minimal in the sense that

$$
\left|\oint_{\gamma^{0}}\langle p, \mathrm{~d} x\rangle\right|=c_{H Z}\left(\Omega_{E}\right) \text {. }
$$


That the result above actually is independent of the choice of Hamiltonian having $\Sigma_{E}$ as energy shell follows from the following property of regular energy shells:

Proposition 16. Let $H$ and $K$ be two time-independent Hamiltonians. Suppose that $E$ and $F$ are regular values of the Hamiltonians $H$ and $K$, respectively, and that

$$
\Sigma=\{z ; H(z)=E\}=\{z ; K(z)=F\} .
$$

Then $H$ and $K$ have the same trajectories on $\Sigma$ (and, in particular, the same periodic orbits).

Proof: The idea underlying the proof is simple: since $E$ and $F$ are regular values, the normals $\partial_{z} H$ and $\partial_{z} K$ to $\Sigma$ do not vanish, and are thus proportional, and so are the Hamilton vector fields $X_{H}=J \partial_{z} H$ and $X_{K}=J \partial_{z} K$; they must thus have the same trajectories, with different parametrizations. In fact, the symplectic orthogonal to the tangent space to $\Sigma$ at every point is a well-defined field of 1directions. This field defines a foliation in lines of the hypersurface $\Sigma$. The leaves of that foliation are the orbits of the vector fields; the result follows.

\section{Acknowledgements}

This work has been partially supported by a grant of the Swedish Royal Academy.

\section{References}

[1] Abraham R. and Marsden J., Foundations of Mechanics, Addison-Wesley, 1978.

[2] Arnold V., Small Denominators and Problems of Stability of Motion in Classical and Celestial Mechanics, Russian Mathematical Surveys 18 (1963) 85-191 [also in Hamiltonian Dynamical Systems, R. MacKay and J. Meiss (Eds.), Adam Hilger, IOP, pp. 260-366].

[3] Berry M., The Adiabatic Limit and the Semiclassical Limit, J. Phys. A: Math. Gen. 17 (1984) 15-27.

[4] Berry M., Classical Adiabatic Angles and Quantal Adiabatic Phase, J. Phys. A: Math. Gen. 18 (1985) 1225-1233.

[5] Berry M. and Wilkinson, M ., Diabolical Points in the Spectra of Triangles, Proc. R. Soc. London A 392 (1984) 15-43. 
[6] Berry M. and Tabor M., Closed Orbits and the Regular Bound Spectrum, Proc. R. Soc. Lond. A. 349 (1976) 101-123.

[7] Bliokh K., Geometric Amplitude, Adiabatic Invariants, Quantization, and Strong Stability of Hamiltonian Systems, J. Math. Phys. 43 (2002) 25-42.

[8] Brown R., Ott E. and Grebogi C., The Goodness of Ergodic Adiabatic Invariants, Journal of Statistical Physics. 49 (1987) 511-550.

[9] Ehrenfest P., Adiabatische invarianten und quantentheorie, Ann. der Physik 51 (1916) 327-352.

[10] Ekeland I. and Ghoussoub N., Selected New Aspects of the Calculus of Variations in the Large, Bull. Amer. Math. Soc. 39 (2002) 207-265.

[11] Ekeland I. and Hofer H., Symplectic Topology and Hamiltonian Dynamics I \& II, Math. Z. 200 (1990) 355-378 and 203 553-567.

[12] de Gosson M., The Principles of Newtonian and Quantum Mechanics, with a Foreword by Basil Hiley, Imperial College Press, London, 2001.

[13] de Gosson M., The Symplectic Camel and Phase Space Quantization, J. Phys. A: Math. Gen. 34 (2001) 10085-10096.

[14] de Gosson M., The 'Symplectic Camel Principle' and Semiclassical Mechanics, J. Phys. A: Math. Gen. 35 (2002) 6825-6851.

[15] Gromov M. Pseudoholomorphic Curves in Symplectic Manifolds, Invent. Math. 82 (1985) 307-347.

[16] Gutzwiller M., Chaos in Classical and Quantum Mechanics, Springer, New York, 1990.

[17] Hannay J., Angle Variable Holonomy in Adiabatic Excursion of an Integrable Hamiltonian, J. Phys. A: Math. Gen. 18 (1985) 221-230.

[18] Hofer H., Hamiltonian Dynamics, Variational Principles and Symplectic Invariants, Preprint, Courant Institute, 2001.

[19] Hofer H. and Zehnder E., Symplectic Invariants and Hamiltonian Dynamics, Birkhäuser Advanced Texts, 1994.

[20] Jammer M., The Conceptual Development of Quantum Mechanics, Inst. Series in Pure and Appl. Physics, McGraw-Hill, 1966.

[21] Koiller J., Classical Adiabatic Angles for Slowly Moving Mechanical Systems, AMS, Contemporary Math. 97, (1989) 159-185.

[22] Libermann P. and Marle C.-M., Symplectic Geometry and Analytical Mechanics, D. Reidel, Dordrecht, 1987.

[23] Marsden J. and Ratiu T., Introduction to Mechanics and Symmetry, Springer, New York, 1994. 
[24] McDuff D. and Salamon D., Symplectic Topology, Oxford Univ, Press, Oxford, 1998.

[25] Montgomery R., The Connection Whose Holonomy is the Classical Adiabatic Angles of Hannay and Berry and Its Generalization to the NonIntegrable Case, Commun. Math. Phys. 120 (1988) 254-270.

[26] Ott E., Goodness of Ergodic Adiabatic Invariants, Phys. Rev. Lett. 42 (1979) 1628-1631.

[27] Polterovich E., The Geometry of the Group of Symplectic Diffeomorphisms, Lectures in Mathematics, ETH Lecture Notes, Birkhäuser, Basel, 2001.

[28] Sugita A., Semiclassical Trace Formulas in Terms of Phase Space Path Integrals, Ann. Phys. 288 (2001) 277-324.

[29] Verhulst F., Nonlinear Differential Systems and Dynamical Systems, Springer, New York, 1990.

[30] Weinstein A., Connections of Berry and Hannay Type for Moving Lagrangian Submanifolds, Adv. in Math. 82 (1990) 133-159.

Maurice A. de Gosson

Blekinge Institute of Technology

Department of Mathematics

SE - 37179 Karlskrona

Sweden 